\title{
Cytidine-5-diphosphocholine reduces microvascular permeability during experimental endotoxemia
}

\author{
Karsten Schmidt ${ }^{*+}$, Jochen Frederick Hernekamp ${ }^{2 \dagger}$, Miriam Doerr $^{3}$, Aleksandar R. Zivkovic ${ }^{1}$, Thorsten Brenner ${ }^{1}$, \\ Andreas Walther ${ }^{4}$, Markus A. Weigand ${ }^{1}$ and Stefan Hofer ${ }^{1}$
}

\begin{abstract}
Background: Microvascular permeability and leukocyte adhesion are pivotal mechanisms in sepsis pathophysiology contributing to the development of shock and mortality. No effective pharmacological therapy is currently available to restore microvascular barrier function in sepsis. Cholinergic mediators have been demonstrated to exert anti-inflammatory effects during inflammation. Cytidine-5-diphosphocholine (CDP-choline) is an extensively studied cholinergic drug due to its brain protective characteristics in cerebrovascular diseases. This study evaluated the effect of CDP-choline on microvascular permeability and leukocyte adhesion during endotoxemia.

Methods: Macromolecular leakage, leukocyte adhesion, and venular wall shear rate were examined in mesenteric postcapillary venules of rats by using intravital microscopy (IVM). Lipopolysaccharide (LPS) $(4 \mathrm{mg} / \mathrm{kg} / \mathrm{h}$ ) or equivalent volumes of saline were continuously infused following baseline IVM at 0 min. IVM was repeated after 60 and $120 \mathrm{~min}$ in endotoxemic and nonendotoxemic animals. CDP-choline (100 mg/kg) was applied as an i.v. bolus. Animals received either saline alone, CDP-choline alone, CDP-choline 10 min before or 30 min after LPS administration, or LPS alone. Due to nonparametric data distribution, Wilcoxon test and Dunn's multiple comparisons test were used for data analysis. Data were considered statistically significant at $p<0.05$.
\end{abstract}

Results: Treatment with LPS alone significantly increased microvascular permeability and leukocyte adhesion and decreased venular wall shear rate. CDP-choline significantly reduced microvascular permeability in animals treated with LPS. Leukocyte adhesion and venular wall shear rate were not affected by CDP-choline during endotoxemia.

Conclusion: CDP-choline has a protective effect on microvascular barrier function during endotoxemia. Considering the excellent pharmacologic safety profile of CDP-choline, its use could be an approach for the treatment of capillary leakage in sepsis.

Keywords: Endotoxemia, CDP-choline, Endothelial dysfunction, Microvascular permeability, Intravital microscopy

\section{Background}

Sepsis is a serious problem in critical care medicine with significant mortality [1] directly associated to microcirculatory alterations [2]. Microcirculatory alterations are early indicators of tissue injury prior to septic organ failure, characterized by the functional breakdown of microvascular perfusion with concomitant tissue edema

\footnotetext{
* Correspondence: karsten.schmidt@med.uni-heidelberg.de

${ }^{\dagger}$ Equal contributors

'Department of Anesthesiology, Heidelberg University Hospital, Im Neuenheimer Feld 110, 69120 Heidelberg, Germany

Full list of author information is available at the end of the article
}

formation. Progressive edema formation due to increased endothelial permeability leads to impaired tissue oxygenation, which precedes organ damage and shock in sepsis.

Inflammatory endothelial cell activation and leukocyteendothelial interactions can be positively influenced by cholinergic mediators [3, 4]. This beneficial cholinergic anti-inflammatory effect on endothelial function can be attributed to the activation of anti-inflammatory neuroimmunological mechanisms described by Tracey et al. $[5,6]$. The inflammatory reflex and the cholinergic anti-inflammatory pathway (CAP) modulate the magnitude of the innate immune response by limiting pro- 
inflammatory processes to a non-toxic range, thereby minimizing tissue injury $[5,6]$. CAP-mediated antiinflammatory signals result in the release of acetylcholine (ACh), which interacts with innate immune cells that express the nicotinic acetylcholine receptor subunit $\alpha 7$ $(\alpha 7 \mathrm{nAChR})$. Intracellular $\alpha 7 \mathrm{nAChR}$ signal transduction inhibits the transcription of pro-inflammatory genes $[5,6]$. Human and rat endothelial cells express $\alpha 7 \mathrm{nAChR}$, identifying the endothelium as a target for anti-inflammatory cholinergic mediators $[3,7]$.

Cytidine-5-diphosphocholine (CDP-choline) has been studied for its brain protective properties in cerebrovascular and neurodegenerative diseases in several clinical trials, and has shown an excellent drug safety profile [8-10].

Locally administered CDP-choline reduced TNF alpha production and tissue edema in a carrageenan-induced inflammatory pain model dependent on the presence of inflammation and non-neuronal $\alpha 7 \mathrm{nAChR}$ [11]. We hypothesized that CDP-choline could reverse sepsisinduced microcirculatory alterations. Therefore this study determined the effects of CDP-choline on microvascular permeability and leukocyte-endothelial interactions during experimental endotoxemia.

\section{Methods}

The general aspects of the materials and methods used have been described in detail in previous publications $[4,12]$.

\section{Anesthesia and animal preparation}

The Governmental Animal Protection Committee approved the experimental protocol used in this investigation (project licence number 35-9185.81/G-123/10). Male Wistar rats $(n=39$, randomized to study groups, 250-300 g body weight; Janvier; St Berthevin, France) were maintained in an animal facility with a 12-h lightdark cycle and housed in stainless steel cages in a temperature-and humidity-controlled room. Standard diet and water were available ad libitum.

Anesthesia was induced with $60 \mathrm{mg} / \mathrm{kg}$ pentobarbital i.p. (Narcoren', Merial GmbH, Hallbergmoos, Germany). Anesthesia was maintained with repeated injections of $10 \mathrm{mg} / \mathrm{kg}$ pentobarbital i.v.. The left carotid artery was cannulated for continuous real time monitoring of mean arterial pressure (MAP $[\mathrm{mmHg}]$ ) and heart rate (HR $\left.\left[\mathrm{min}^{-1}\right]\right)$ using a computerized non-commercial small animal monitoring system (Small Animal Monitoring Vs. 1.2.6.3, Exp. Chirurgie, Heidelberg 2012). The right jugular vein was cannulated for administrating test substances. Temperature was measured with a rectal thermistor probe and maintained at $37{ }^{\circ} \mathrm{C}$. A segment of the ileum was gently exteriorized through a mid-line abdominal incision and draped over a clear glass pedestal for intravital microscopy examination. Exposed tissues were continuously superfused with buffered (pH 7.4) and thermostat-controlled Ringer solution $\left(37^{\circ} \mathrm{C}\right)$.

A stock solution of LPS (Lipopolysaccharide, Escherichia coli 026:B6, Sigma-Aldrich Chemie GmbH, Steinheim, Germany) was prepared by dissolving LPS in saline to a concentration of $5 \mathrm{mg} / \mathrm{ml}$. The solution was stored in a glass container at $5{ }^{\circ} \mathrm{C}$. The stock solution was diluted in saline to the appropriate concentration for experiments. Infusions of LPS $(4 \mathrm{mg} / \mathrm{kg} / \mathrm{h})$ or equivalent volumes of saline solution were administered via the jugular vein. The LPS dosage of $4 \mathrm{mg} / \mathrm{kg} / \mathrm{h}$ was identified in pilot experiments as the LPS dosage without pronounced hypotension and a significant inflammatory endothelial activation (macromolecular leakage, leukocyte-endothelial interactions) compared to nonendotoxemic animals.

Cytidine-5-diphosphocholine (CDP-choline, SigmaAldrich Chemie GmbH, Steinheim, Germany) was diluted in saline to the appropriate concentration for each rat and was injected i.v. according to the experimental protocol (Fig. 1).

For measurement of erythrocyte velocity fluorescentlabeled erythrocytes from donor rats were injected $10 \mathrm{~min}$ before baseline measurements $(0.5 \mathrm{~mL} / \mathrm{kg}$ body weight; hematocrit $50 \%$; labeled with a red fluorescent cell linker kit (PKH26-GL; Sigma Chemical, Deisenhofen, Deisenhofen, Germany).

To quantify albumin leakage across mesenteric venules, $50 \mathrm{mg} / \mathrm{kg}$ of fluorescein isothiocyanate-labeled bovine albumin (FITC-albumin; Sigma Chemicals, Deisenhofen, Germany) was injected 10 min before baseline measurements.

\section{Intravital microscopy}

Intravital fluorescence microscopy (IVM) was performed at 0,60 , and $120 \mathrm{~min}$ (Fig. 1). Selected post-capillary venules were observed with a specially designed microscope (Orthoplan, Leica, Wetzlar, Germany) equipped with a 40-fold water immersion objective (Achroplan 40/0.75 W; Zeiss, Jena, Germany).

Images were recorded and digitized with a digital camera (TypPS/DX4-285FW, Kappa opto-electronics $\mathrm{GmbH}$, Gleichen, Germany) equipped with a capturing software (Streampix 5.3.0, Norpix Inc., Montreal, Canada).

Image (NIH, Bethesda, MD) and Histo (Histo, Version 3.0.2.4, Exp. Chirurgie, Uniklinik Heidelberg 2011) were used for offline image analysis.

The analysis software was calibrated to the aforementioned microscope/camera setting yielding a pixel spacing of $3.25 \mathrm{pixel} / \mu \mathrm{m}$ in effective magnification. A fluorescence gray scale from black to white (gray levels ranging from 0 [black] to 255 [white]) with fixed brightness and contrast levels was used for recording and analysis. 


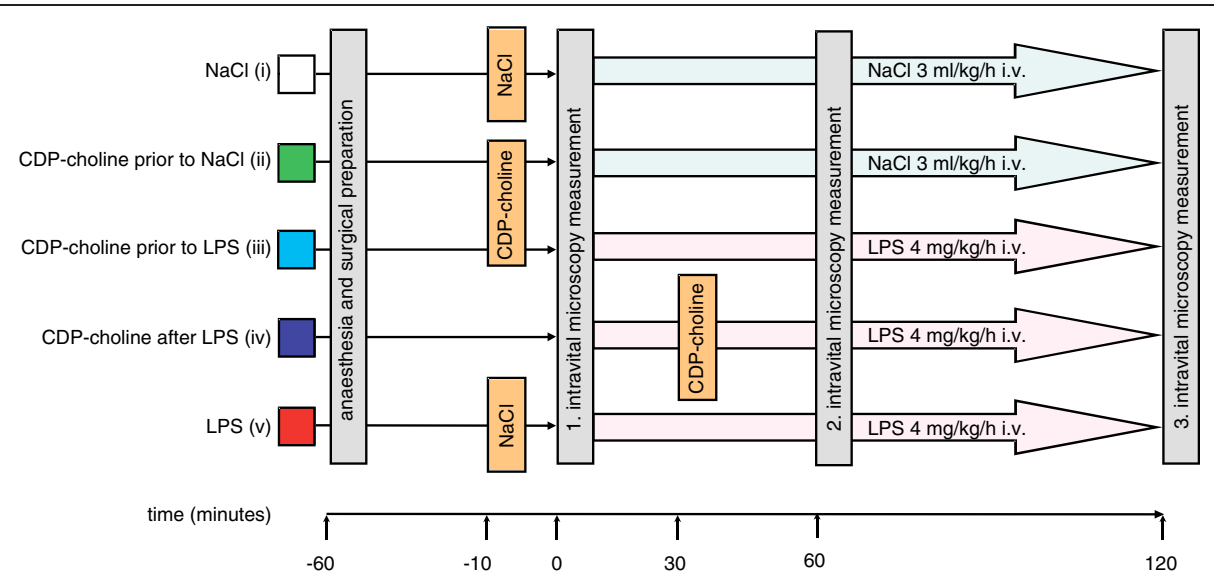

Fig. 1 Experimental protocol. Intravital microscopic measurements (IVM) were performed at 0-, 60-, and 120- min in endotoxemic and nonendotoxemic animals following a stabilization period after surgical preparation. LPS (4 mg/ $\mathrm{kg} / \mathrm{h}$ ) or an equivalent volume of saline was continuously infused starting directly after baseline IVM at $0 \mathrm{~min}$. CDP-choline $(100 \mathrm{mg} / \mathrm{kg})$ was applied as an i.v. bolus in treatment groups. All administered fluids were calculated to guarantee that all animals received equal amounts of intravenous fluids. The color and number code of the experimental groups introduced in this figure is used in all other figures

\section{Quantification of macromolecular leakage}

The recorded fluorescent images were digitized and the gray levels reflecting fluorescent intensity were measured within the venule under study (iv) as well as in an equal and contiguous area of the perivenular interstitium (ii). Macromolecular leakage was determined as ii/iv ratio (arbitrary units).

\section{Leukocyte-endothelial interactions}

The behavior of leukocytes was visualized using transillumination microscopy. Adherent leukocytes were defined as cells that did not move or detach from the endothelial wall for a period of $30 \mathrm{sec}$ and were counted offline during playback of the recorded videos. Leukocyte adherence was expressed as the number of cells per square millimetre of vessel surface as calculated from the diameter and length of the vessel segment studied.

\section{Measurement of venular wall shear rate}

Mean red blood cell velocities $\left(\mathrm{V}_{\mathrm{RBC}}\right)$ in single unbranched post-capillary venules were calculated by averaging the velocities of 20-30 individual erythrocytes. The distance through which a labeled erythrocyte travelled within two subsequent video frames was divided by the video frame time interval. Venular wall shear rate was calculated on the basis of the Newtonian definition $\left(\gamma=8\left(V_{R B C} / D_{v}\right)\right)$, using the measured vessel diameters of the observed postcapillary venules.

\section{Experimental protocol}

The experimental protocol is illustrated in Fig. 1. The color code of the experimental groups introduced in Fig. 1 is used in the results section for Fig. 2 and Fig. 4 as well.
Anaesthesia and surgical preparation was identical for all animals. A 45-min stabilization period followed exteriorization of the mesentery. Animals were randomized to the respective treatment groups $10 \mathrm{~min}$ before baseline intravital microscopic measurements (IVM) at $0 \mathrm{~min}$. IVM were performed at 0,60 , and $120 \mathrm{~min}$ in endotoxemic and nonendotoxemic animals. Macrohemodynamic parameters (single values; MAP and HR) were documented at these time points. LPS $(4 \mathrm{mg} / \mathrm{kg} / \mathrm{h})$ or an equivalent volume of saline was continuously infused starting directly after baseline IVM at $0 \mathrm{~min}$. CDP-choline $(100 \mathrm{mg} / \mathrm{kg})$ was applied as an i.v. bolus in treatment groups. All administered fluids were calculated to guarantee that all animals received equal amounts of intravenous fluids.

After randomization animals $[n=39]$ received saline alone in the $\mathrm{NaCl}$ (i) [ $n=8]$ group, CDP-choline $10 \mathrm{~min}$ prior to saline infusion (ii) $[n=6]$, CDP-choline $10 \mathrm{~min}$ prior to LPS administration (iii) $[n=8]$, CDP-choline 30 min after LPS administration (iv) $[n=9]$, and LPS alone (v) $[n=8]$ in the endotoxemia group.

At baseline IVM inclusion criteria for postcapillary venules were defined as a diameter of 25-35 $\mu \mathrm{m}$, no more than 5 adherent leukocytes per $100 \mu \mathrm{m}$ vessel length, no blood flow stasis, no lymph vessels or mesenterial fat tissue in the immediate vicinity of the observed vessel segment and at least one free vessel wall without concomitant or branching vessels.

In total 16 venules in the $\mathrm{NaCl}$ (i), 17 venules in the CDP-choline (ii), 22 venules in the CDP-choline (iii), 23 venules in the CDP-choline (iv), and 17 venules in the LPS (vi) groups fulfilled the criteria stated above and were recorded and analyzed. 


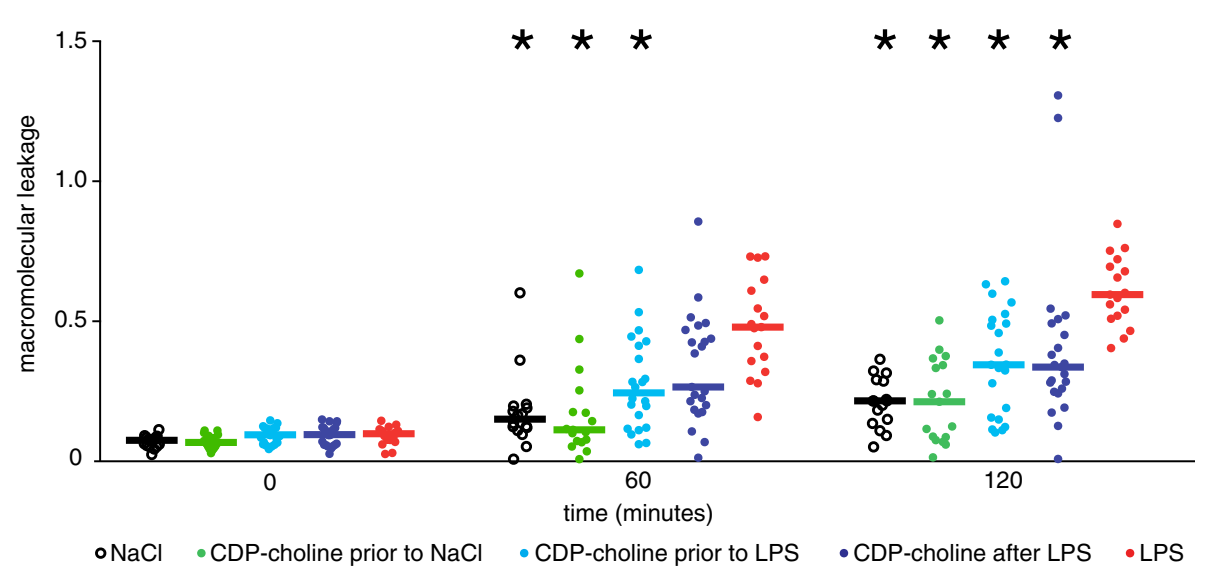

Fig. 2 Effect of CDP-choline administration on macromolecular leakage during endotoxemia. Macromolecular leakage is expressed as ratio of perivenular to venular fluorescence intensity in arbitrary units. CDP-choline reduced microvascular permeability in postcapillary venules during endotoxemia. These results indicate that CDP-choline has a protective effect on microvascular permeability. Scatterplots with medians are displayed. *Significant difference vs. LPS (v). Medians with interquartile range (Q1-Q3) and $p$ values are presented in Table 1

\section{Statistical analysis}

The main outcome parameter for this study was the perivenular macromolecular leakage around postcapillary venules. To evaluate the effect of CDP-choline on microvascular alterations during endotoxemia, all IVMgenerated microvascular data are compared to the LPS group unless otherwise stated. The D'Agostino \& Pearson omnibus normality test was applied to check for normal distribution. Due to nonparametric data distribution, nonparametric methods for evaluation were used (Wilcoxon test for differences within groups and Dunn's multiple comparisons test for differences between groups with multiplicity adjusted $\mathrm{p}$ values). Data were considered statistically significant at $p<0.05$.

The results are presented in Tables 1, 2, 3 as median with interquartile range (Q1-Q3). Significant adjusted p-values of the comparisons with the LPS group and significant p-values of the comparisons with the baseline IVM measurement at $0 \mathrm{~min}$ are included in the tables.
Statistical analysis was performed using GraphPad Prism version 6.0 for Mac OS X, GraphPad Software, San Diego California USA, www.graphpad.com.

\section{Results}

\section{Macromolecular leakage}

The baseline IVM measurement at $0 \mathrm{~min}$ revealed no significant differences in macromolecular leakage between all experimental groups. Macromolecular leakage increased significantly in all groups over $120 \mathrm{~min}$ compared to the respective baseline measurements with the highest rise of plasma extravasation in the LPS (v) group. Infusion of LPS (v) caused a significant increase in macromolecular leakage compared to the $\mathrm{NaCl}$ (i) group at 60 and $120 \mathrm{~min}$. After $120 \mathrm{~min}$ all groups treated with CDP-choline showed a significantly reduced macromolecular leakage compared to the LPS (v) group. Table 1 summarizes the results of the IVM measurements of macromolecular leakage. Figure 2 illustrates the

Table 1 Summarized results of macromolecular leakage

\begin{tabular}{|c|c|c|c|c|c|c|c|c|c|c|c|c|}
\hline \multirow[b]{3}{*}{$\begin{array}{l}\text { Macromolecular leakage } \\
\text { [arbitrary units] }\end{array}$} & & \multicolumn{11}{|c|}{ Time after induction of endotoxemia } \\
\hline & & \multicolumn{3}{|l|}{$0 \mathrm{~min}$} & \multicolumn{4}{|l|}{$60 \mathrm{~min}$} & \multicolumn{4}{|l|}{$120 \mathrm{~min}$} \\
\hline & & Median & IQR (Q1-Q3) & $*$ & Median & IQR (Q1-Q3) & ${ }^{* 1}$ & $\#^{2}$ & Median & IQR (Q1-Q3) & $*$ & $\#$ \\
\hline $\mathrm{NaCl}$ & (i) & 0.075 & $0.05-0.09$ & $n s^{3}$ & 0.15 & $0.11-0.2$ & $<0.0001$ & 0.0002 & 0.22 & $0.14-0.29$ & $<0.0001$ & $<0.0001$ \\
\hline CDP-choline prior to $\mathrm{NaCl}$ & (ii) & 0.067 & $0.05-0.09$ & ns & 0.11 & $0.07-0.21$ & $<0.0001$ & 0.0054 & 0.21 & $0.08-0.36$ & $<0.0001$ & 0.0008 \\
\hline CDP-choline prior to LPS & (iii) & 0.095 & $0.07-0.12$ & ns & 0.24 & $0.12-0.42$ & 0.0141 & $<0.0001$ & 0.35 & $0.15-0.51$ & 0.0019 & $<0.0001$ \\
\hline CDP-choline after LPS & (iv) & 0.095 & $0.06-0.12$ & ns & 0.27 & $0.18-0.47$ & ns & $<0.0001$ & 0.34 & $0.25-0.49$ & 0.0022 & $<0.0001$ \\
\hline LPS & (v) & 0.099 & $0.07-0.11$ & & 0.48 & $0.34-0.63$ & & $<0.0001$ & 0.6 & $0.51-0.71$ & & $<0.0001$ \\
\hline
\end{tabular}

$1 *$ significant adjusted $\mathrm{p}$ value vs. LPS (v)

2 \# significant $p$ value vs. 0 min

${ }^{3}$ ns: not significant 
Table 2 Summarized numbers of adherent leukocytes

\begin{tabular}{|c|c|c|c|c|c|c|c|c|c|c|c|c|}
\hline \multirow{3}{*}{\multicolumn{2}{|c|}{ Adherent leukocytes [cells/ $\mathrm{mm}^{2}$ ] }} & \multicolumn{11}{|c|}{ Time after induction of endotoxemia } \\
\hline & & \multicolumn{3}{|c|}{$0 \min$} & \multicolumn{4}{|l|}{$60 \mathrm{~min}$} & \multicolumn{4}{|l|}{$120 \mathrm{~min}$} \\
\hline & & \multirow{2}{*}{$\begin{array}{l}\text { Median } \\
140\end{array}$} & \multirow{2}{*}{$\frac{\text { IQR (Q1-Q3) }}{59-218}$} & \multirow{2}{*}{$\frac{{ }^{*}}{n s^{3}}$} & \multirow{2}{*}{$\begin{array}{l}\text { Median } \\
172\end{array}$} & \multirow{2}{*}{$\frac{\text { IQR (Q1-Q3) }}{85-201}$} & \multirow{2}{*}{$\begin{array}{l}* 1 \\
0.0139\end{array}$} & \multirow{2}{*}{$\frac{\#^{2}}{\mathrm{~ns}}$} & \multirow{2}{*}{$\begin{array}{l}\text { Median } \\
136\end{array}$} & \multirow{2}{*}{$\frac{\text { IQR (Q1-Q3) }}{74-216}$} & \multirow{2}{*}{$\begin{array}{l}* 0.0001 \\
<0.04\end{array}$} & \multirow{2}{*}{ \# } \\
\hline $\mathrm{NaCl}$ & (i) & & & & & & & & & & & \\
\hline CDP-choline prior to $\mathrm{NaCl}$ & (ii) & 206 & $75-268$ & ns & 204 & $108-251$ & ns & ns & 220 & $147-337$ & 0.015 & ns \\
\hline CDP-choline prior to LPS & (iii) & 204 & $165-250$ & ns & 243 & $169-375$ & ns & 0.0175 & 322 & $234-423$ & ns & $<0.000$ \\
\hline CDP-choline after LPS & (iv) & 239 & $128-271$ & ns & 305 & $232-351$ & ns & $<0.0001$ & 342 & $226-418$ & ns & 0.0003 \\
\hline LPS & (v) & 193 & $118-280$ & & 267 & $193-358$ & & 0.0038 & 403 & $224-562$ & & $<0.0001$ \\
\hline
\end{tabular}

$1 *$ significant adjusted $p$ value vs. LPS (v)

2 \# significant $\mathrm{p}$ value vs. 0 min

${ }^{3}$ ns: not significant

observed effect of CDP-choline on macromolecular leakage and displays the significant differences to the LPS (v) group. Representative IVM images are shown in Fig. 3.

\section{Leukocyte adherence}

No significant differences were observed between all groups at the beginning of the experiment. The number of adhering leukocytes increased significantly over 120 min in all LPS treated groups (iii, iv, v) when compared to the respective baseline measurement. Leukocyte adhesion was significantly increased in the LPS group at 60 and 120 min compared to the $\mathrm{NaCl}$ (i) group and after 120 min compared to the nonendotoxemic CDPcholine (ii) group. No significant difference between the LPS (v) group and the endotoxemic CDP-choline (iii, iv) groups was observed during the experiment. CDPcholine administration without endotoxemia (ii) did not result in a significant increase in adhering leukocytes. In endotoxemic animals treated with CDP-choline (iii, iv) leukocyte adherence increased significantly compared to the baseline measurement during the experiment. Table 2 shows the summarized numbers of adherent leukocytes. Figure 4 illustrates the observed effect of CDP-choline leukocyte adhesion and displays the significant differences to the LPS (v) group.

Table 3 Summarized macro- and microhemodynamic parameters

\begin{tabular}{|c|c|c|c|c|c|c|c|c|c|c|c|c|}
\hline \multirow{3}{*}{\multicolumn{2}{|c|}{ Mean arterial pressure $[\mathrm{mmHg}]$}} & \multicolumn{11}{|c|}{ Time after induction of endotoxemia } \\
\hline & & \multicolumn{3}{|l|}{$0 \mathrm{~min}$} & \multicolumn{4}{|l|}{$60 \mathrm{~min}$} & \multicolumn{4}{|l|}{$120 \mathrm{~min}$} \\
\hline & & Median & IQR (Q1-Q3) & * & Median & IQR (Q1-Q3) & $*^{1}$ & $\#^{2}$ & Median & IQR (Q1-Q3) & * & \# \\
\hline $\mathrm{NaCl}$ & (i) & 117 & $100-126$ & $n s^{3}$ & 131 & $125-137$ & 0.0037 & ns & 133 & $118-145$ & 0.0386 & ns \\
\hline CDP-choline prior to $\mathrm{NaCl}$ & (ii) & 108 & $101-118$ & ns & 117 & $93-132$ & ns & ns & 133 & 104-139 & ns & ns \\
\hline CDP-choline prior to LPS & (iii) & 105 & $97-123$ & ns & 105 & $101-114$ & ns & ns & 113 & $60-117$ & ns & ns \\
\hline CDP-choline after LPS & (iv) & 106 & $94-120$ & ns & 105 & $89-120$ & ns & ns & 114 & $100-122$ & ns & ns \\
\hline LPS & (v) & 103 & 89-109 & & 99 & 89-104 & & ns & 106 & $97-112$ & & ns \\
\hline Heart rate $\left[\mathrm{min}^{-1}\right]$ & & Median & IQR (Q1-Q3) & * & Median & IQR (Q1-Q3) & * & $\#$ & Median & IQR (Q1-Q3) & * & \# \\
\hline $\mathrm{NaCl}$ & (i) & 322 & $317-344$ & ns & 371 & $332-403$ & 0.0037 & 0.0002 & 395 & $326-441$ & 0.0327 & $<0.0001$ \\
\hline CDP-choline prior to $\mathrm{NaCl}$ & (ii) & 358 & $338-411$ & ns & 408 & $400-473$ & ns & 0.0313 & 433 & $428-470$ & ns & 0.0313 \\
\hline CDP-choline prior to LPS & (iii) & 375 & $351-456$ & ns & 475 & $447-566$ & ns & 0.0156 & 449 & $441-493$ & ns & 0.0313 \\
\hline CDP-choline after LPS & (iv) & 353 & $287-380$ & ns & 381 & $326-432$ & ns & 0.0117 & 445 & $358-476$ & ns & 0.0039 \\
\hline LPS & (v) & 340 & $320-353$ & & 410 & $391-436$ & & 0.0078 & 491 & 435-516 & & 0.0078 \\
\hline Venular wall shear rate $\left[\mathrm{s}^{-1}\right]$ & & Median & IQR (Q1-Q3) & * & Median & IQR (Q1-Q3) & ${ }^{*}$ & $\#$ & Median & IQR (Q1-Q3) & * & $\#$ \\
\hline $\mathrm{NaCl}$ & (i) & 400 & $352-575$ & ns & 505 & $413-642$ & ns & 0.0006 & 444 & $368-573$ & ns & ns \\
\hline CDP-choline prior to $\mathrm{NaCl}$ & (ii) & 550 & $427-653$ & ns & 420 & $356-614$ & ns & ns & 302 & $230-631$ & ns & 0.0395 \\
\hline CDP-choline prior to LPS & (iii) & 436 & $367-567$ & ns & 362 & $274-553$ & ns & 0.0229 & 311 & $120-497$ & ns & 0.0009 \\
\hline CDP-choline after LPS & (iv) & 503 & $407-582$ & ns & 397 & $321-505$ & ns & 0.0301 & 443 & $281-590$ & ns & 0.0254 \\
\hline LPS & (v) & 499 & $405-622$ & & 378 & $251-523$ & & ns & 348 & $285-493$ & & 0.0026 \\
\hline
\end{tabular}

$1 *$ significant adjusted $p$ value vs. LPS (v)

2 \# significant $p$ value vs. 0 min

${ }^{3}$ ns: not significant 


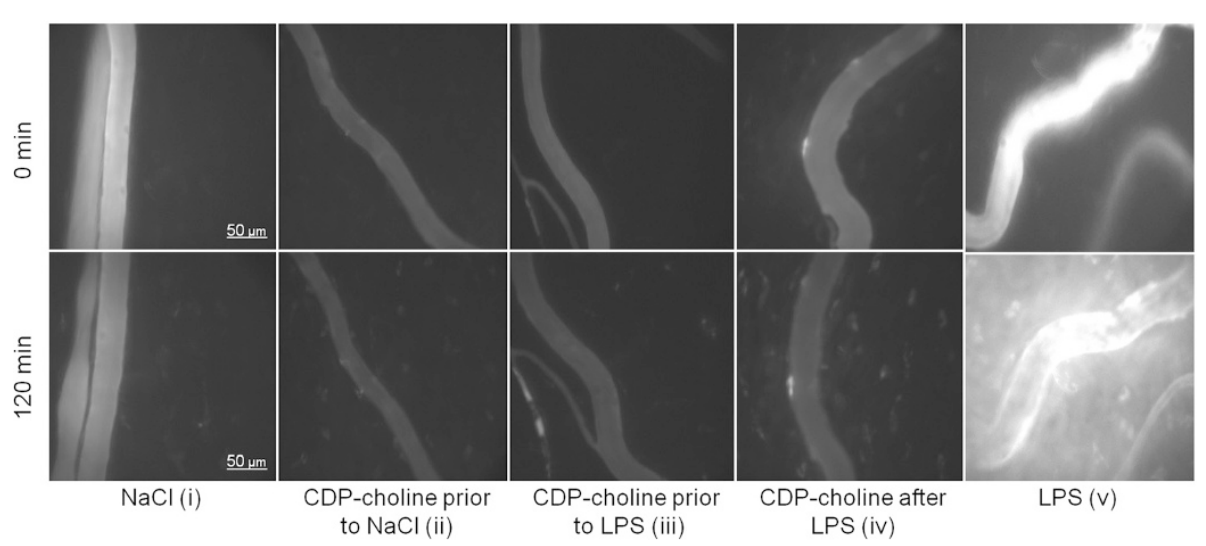

Fig. 3 IVM images demonstrating the CDP-choline effect on microvascular permeability during endotoxemia. Fluorescent IVM images show postcapillary venules recorded at 0 min (upper panels) and $120 \mathrm{~min}$ after the treatment (lower panels). LPS administration alone (v) induces distinctive macromolecular leakage compared to the baseline measurement at 0 min and to the $\mathrm{NaCl}$ group (i). Note the effect of CDP-choline on reducing macromolecular leakage in endotoxemic animals (iii, iv) compared to the LPS group (v)

\section{Macro- and microhemodynamic changes}

There were no significant differences between all groups in the macro- and microhemodynamic parameters at the beginning of the experiment. The mean arterial pressure differed significantly between the $\mathrm{NaCl}$ (i) - and LPS (v) group at $60 \mathrm{~min}$ and at $120 \mathrm{~min}$. No significant change in mean arterial pressure was observed within the groups. All groups showed a significant increase in heart rate over $120 \mathrm{~min}$ compared to the baseline measurement. Venular wall shear rate decreased significantly within the LPS group over $120 \mathrm{~min}$. The venular wall shear rate decreased significantly after 120 min within all CDP-choline groups. The observed macro- and microhemodynamic changes are summarized in Table 3 including the significant changes compared to the IVM measurement at $0 \mathrm{~min}$ and to the LPS (v) group.

\section{Discussion}

This study examined the effect of Cytidine-5diphosphocholine (CDP-choline) on microcirculatory alterations during endotoxemia. LPS administration significantly increased microvascular permeability and leukocyte adherence and decreased venular wall shear rate in postcapillary venules. CDP-choline significantly reduced macromolecular leakage when applied before and after the start of the LPS infusion. In endotoxemic animals CDP-choline did not affect the number of

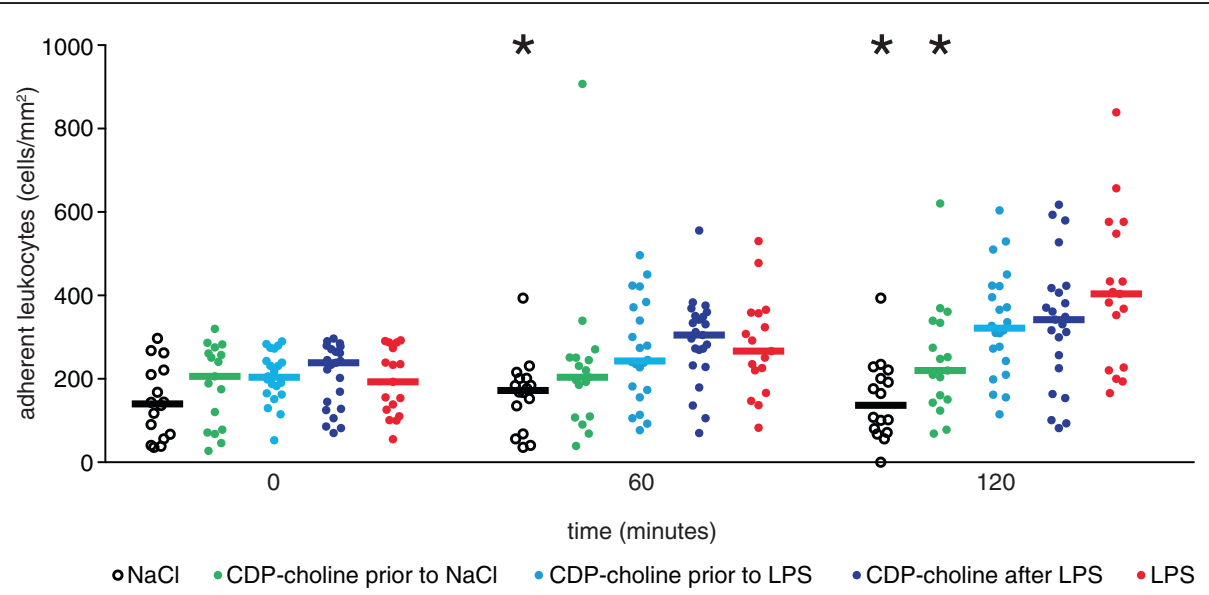

Fig. 4 Effect of CDP-choline administration on leukocyte adherence during endotoxemia. CDP-choline had no effect on the number of adhering leukocytes during endotoxemia. Scatterplots with medians are displayed. * Significant difference vs. LPS (v). Medians with interquartile range (Q1-Q3) and $p$ values are presented in Table 2 
adherent leukocytes and the reduced venular wall shear rate observed after LPS treatment.

The key result of this study is that CDP-choline has a protective effect on microvascular permeability during experimental endotoxemia.

Non-neuronal cholinergic mechanisms are fundamental processes for the fine-tuning of endothelial hemoeostasis [13]. Both direct and indirect cholinergic stimulation exhibit anti-inflammatory effects on endothelial activation and leucocyte-endothelial interactions [3, 4, 14]. Proinflammatory endothelial cell activation can be suppressed by the activation of specific nicotinic acetylcholine receptors like the $\alpha 7 \mathrm{nAChR}[15]$ associated with the cholinergic anti-inflammatory pathway $[5,6,16]$. CDPcholine had a protective effect on microvascular barrier function when applied before and after the onset of endotoxemia in this study. Intravenous administration of CDP-choline increases plasma and brain choline and acetylcholine levels in rats [17, 18]. Choline as a selective natural $\alpha 7 \mathrm{nAChR}$ agonist improves survival in experimental sepsis [19]. Gurun et al. demonstrated that localized administration of CDP-choline reduced tissue edema and TNF- $\alpha$ production in a $\alpha 7 \mathrm{nAChR}$-dependent mechanism [11]. Moreover activation of central cholinergic transmission by CDP-choline has protective $\alpha 7 \mathrm{nAChR}$ - independent effects on myocardial ischemia-reperfusion damage [18].

The observed protective effect of CDP-choline on microvascular permeability is therefore probably attributable to a cholinergic activation of endothelial cells that could be directly mediated by $\alpha 7 \mathrm{nAChR}$ on a cellular level or by central anti-inflammatory cholinergic signals. This protective effect is in line with scientific evidence regarding the anti-inflammatory potential of CDP-choline in other experimental settings [20-23].

There is ample evidence for interdependent leukocytedependent and leukocyte-independent mechanisms affecting endothelial permeability [12, 24]. Leukocyte recruitment can be blocked by $\alpha 7 \mathrm{nAChR}$-dependent cell activation [3].

In contrast to the effect of the centrally acting acetylcholinesterase inhibitor physostigmine, which reduced leukocyte adherence [4], CDP choline did not affect leukocyte adhesion during endotoxemia. This could indicate that leukocyte activation is more affected by centrally mediated cholinesterase inhibition or by more effectively elevating ACh serum levels. CDP-choline and choline are $\alpha 7 \mathrm{nAChR}$ agonists with a lower potency as ACh, possibly explaining the missing effect on the numbers of adherent leukocytes observed in this study. LPS causes shedding of the endothelial glycocalix in rats [25], and inhibition of glycocalyx shedding reduces leukocyteendothelial adhesion [26]. Increased microvascular permeability due to a breakdown of the endothelial glycocalix results in an increased transcapillary escape rate of albumin in experimental sepsis [27]. The decreased macromolecular leakage of FITC-albumin in the endotoxemic CDP-choline treated groups could be interpreted as a stabilization of the endothelial surface layer. A modulation of glycocalix function influencing the interaction between endothelial cells and leukocytes could be an explanation for the observed protective effect of CDP-choline on microvascular permeability paralleled by increased numbers of adherent leukocytes in the CDP-choline treated endotoxemic groups. Further studies focusing on qualitative effects of CDP-choline on leukocyte-endothelial interactions and glycocalix function are required to evaluate the in vivo findings of this study. The increased leukocyte adherence in LPS-treated animals is consistent with the decreased venular wall shear rate induced by endotoxemia [28]. Venular wall shear rate decreased significantly in all CDP-choline treated groups. Interestingly the largest decrease in venular wall shear rate was observed within the nonendotoxemic CDP-choline treated group indicating a direct effect of CDP-choline on the microcirculation without causing increased microvascular permeability and leukocyte adherence. In LPS treated animals CDP-choline treatment had no distinguishable effect on venular wall shear rate. Microvascular flow is impaired during early sepsis and uncoupled from macrovascular function [28]. This uncoupling is clearly demonstrated by the hemodynamic profile of the LPS (v) group. Macrohemodynamic parameters were not distinguishably affected by CDP-choline treatment. It has been demonstrated that CDP-choline restores renal and mesenteric arterial blood flow and hypotension in shock states and prevents death in myocardial ischaemia-reperfusion injury by activating central cholinergic neurotransmission $[17,18,29]$. This indicates that CDP-choline has positive effects on a compromised circulatory system. We did not induce an endotoxemic shock during the $120 \mathrm{~min}$ observation time of this study therefore no final conclusion can be drawn regarding the hemodynamic effects of CDPcholine. Given the heterogeneity of microcirculatory perfusion during sepsis, microvascular changes in the mesenteric postcapillary venules examined may not reflect the microcirculatory situation in other vascular beds. Therefore further studies to evaluate the hemodynamic and microcirculatory effects of CDP-choline during sepsis remain to be done.

\section{Limitations}

We could not quantify plasma cytokines and glycocalyx marker in blood serum samples of the experimental animals due to the interference of the fluorescent dyes used for IVM with the standardized ELISA technology. Therefore, no direct correlation between our IVM results and the effect of CDP-choline on the systemic 
inflammatory response and the degree of glycocalyx shedding could be drawn.

\section{Conclusion}

CDP-choline was effective in reducing LPS-induced microvascular permeability in endotoxemic rats. Leukocyte adherence and venular wall shear rate were not affected by CDP-choline during endotoxemia. Regarding the effect of CDP-choline on leukocyte-endothel interactions and microcirculatory alterations in this study, no final conclusion can be drawn necessitating further studies.

The protective effect on microvascular permeability of CDP-choline in this study may carefully be used as a basis for a clinical approach to stabilize capillary leakage in human sepsis. CDP-choline has been thoroughly studied in clinical trials for its brain protective properties on cerebrovascular and neurodegenerative diseases with an excellent drug safety profile $[8-10,30]$. Considering its anti-inflammatory efficacy CDP-choline is a substance with great potential for clinical sepsis treatment.

\section{Competing interests}

The authors declare that they have no competing interests.

\section{Authors' contributions}

KS and JFH participated in the design of the study and wrote the manuscript. KS supervised the data acquisition and performed the statistical analysis. MD carried out the experiments. ARZ, TB, AW, MAW and SH have been involved in drafting the manuscript and revising it critically for important intellectual content. AW, MAW and SH have given final approval of the version to be published. All authors read and approved the final manuscript.

\section{Acknowledgement}

The authors would like to thank Thomas Bruckner, Dr. sc.hum., Department of Medical Biometry and Informatics, University of Heidelberg, Heidelberg, Germany for assistance in the analysis and interpretation of data. The authors would like to thank Anuradha Gunale, M.D., Department of Anesthesiology, Heidelberg University Hospital, Heidelberg, Germany for revising the manuscript critically.

\section{Funding}

This project was funded by the Foundation Landesbank Baden-Württemberg, Stuttgart, Germany.

\section{Author details}

'Department of Anesthesiology, Heidelberg University Hospital, Im Neuenheimer Feld 110, 69120 Heidelberg, Germany. ${ }^{2}$ Department of Hand, Plastic and Reconstructive Surgery, Burn Center, BG Trauma Center Ludwigshafen, Ludwigshafen, University of Heidelberg, Heidelberg, Germany. ${ }^{3}$ Department of General, Visceral and Transplant Surgery, University of Heidelberg, Heidelberg, Germany. ${ }^{4}$ Department of Anesthesiology, Katharinenhospital, Stuttgart, Germany.

Received: 25 February 2015 Accepted: 8 July 2015

Published online: 01 August 2015

\section{References}

1. Mayr FB, Yende S, Angus DC. Epidemiology of severe sepsis. Virulence. 2014;5(1):4-11

2. De Backer D, Orbegozo Cortes D, Donadello K, Vincent JL. Pathophysiology of microcirculatory dysfunction and the pathogenesis of septic shock. Virulence. 2014:5(1):73-9.

3. Saeed RW, Varma S, Peng-Nemeroff T, Sherry B, Balakhaneh D, Huston J, et al. Cholinergic stimulation blocks endothelial cell activation and leukocyte recruitment during inflammation. J Exp Med. 2005;201(7):1113-23.
4. Peter C, Schmidt K, Hofer S, Stephan M, Martin E, Weigand MA, et al. Effects of physostigmine on microcirculatory alterations during experimental endotoxemia. Shock. 2010;33(4):405-11.

5. Tracey KJ. Physiology and immunology of the cholinergic antiinflammatory pathway. J Clin Invest. 2007;117(2):289-96.

6. Tracey KJ. Reflex control of immunity. Nat Rev Immunol. 2009;9(6):418-28.

7. Moccia F, Frost C, Berra-Romani R, Tanzi F, Adams DJ. Expression and function of neuronal nicotinic ACh receptors in rat microvascular endothelial cells. Am J Physiol Heart Circ Physiol. 2004;286(2):H486-491.

8. Adibhatla RM, Hatcher JF. Cytidine 5'-diphosphocholine (CDP-choline) in stroke and other CNS disorders. Neurochem Res. 2005;30(1):15-23.

9. Saver JL. Citicoline: update on a promising and widely available agent for neuroprotection and neurorepair. Rev Neurol Dis. 2008;5(4):167-77.

10. Clark WM. Efficacy of citicoline as an acute stroke treatment. Expert Opin Pharmacother. 2009;10(5):839-46.

11. Gurun MS, Parker R, Eisenach JC, Vincler M. The effect of peripherally administered CDP-choline in an acute inflammatory pain model: the role of alpha7 nicotinic acetylcholine receptor. Anesth Analg. 2009;108(5):1680-7.

12. Walther A, Weihrauch M, Schmidt W, Gebhard MM, Martin E, Schmidt H. Leukocyte-independent plasma extravasation during endotoxemia. Crit Care Med. 2000;28(8):2943-8.

13. Kirkpatrick CJ, Bittinger F, Unger RE, Kriegsmann J, Kilbinger $H_{4}$ Wessler I. The non-neuronal cholinergic system in the endothelium: evidence and possible pathobiological significance. Jpn J Pharmacol. 2001;85(1):24-8.

14. Silva-Herdade AS, Saldanha C. Effects of acetylcholine on an animal mode of inflammation. Clin Hemorheol Microcirc. 2013;53(1-2):209-16.

15. Chatterjee PK, Al-Abed Y, Sherry B, Metz CN. Cholinergic agonists regulate JAK2/STAT3 signaling to suppress endothelial cell activation. Am J Physiol Cell Physiol. 2009;297(5):C1294-1306.

16. Wang H, Yu M, Ochani M, Amella CA, Tanovic M, Susarla S, et al. Nicotinic acetylcholine receptor alpha7 subunit is an essential regulator of inflammation. Nature. 2003;421(6921):384-8.

17. Savci V, Goktalay G, Cansev M, Cavun S, Yilmaz MS, Ulus IH. Intravenously injected CDP-choline increases blood pressure and reverses hypotension in haemorrhagic shock: effect is mediated by central cholinergic activation. Eur J Pharmacol. 2003;468(2):129-39.

18. Yilmaz MS, Coskun C, Yalcin M, Savci V. CDP-choline prevents cardiac arrhythmias and lethality induced by short-term myocardial ischemia-reperfusion injury in the rat: involvement of central muscarinic cholinergic mechanisms. Naunyn Schmiedebergs Arch Pharmacol. 2008;378(3):293-301.

19. Parrish WR, Rosas-Ballina M, Gallowitsch-Puerta M, Ochani M, Ochani K Yang LH, et al. Modulation of TNF release by choline requires alpha7 subunit nicotinic acetylcholine receptor-mediated signaling. Mol Med. 2008;14(9-10):567-74.

20. Ilcol YO, Yilmaz Z, Cansev M, Ulus IH. Choline or CDP-choline alters serum lipid responses to endotoxin in dogs and rats: involvement of the peripheral nicotinic acetylcholine receptors. Shock. 2009;32(3):286-94.

21. Jambou R, El-Assaad F, Combes V, Grau GE. Citicoline (CDP-choline): What role in the treatment of complications of infectious diseases. Int J Biochem Cell Biol. 2009;41(7):1467-70.

22. Yilmaz Z, IIcol YO, Torun $\mathrm{S}$, Ulus $\mathbb{H}$. Intravenous administration of choline or cdp-choline improves platelet count and platelet closure times in endotoxin-treated dogs. Shock. 2006;25(1):73-9.

23. Yilmaz Z, Ozarda Y, Cansev M, Eralp O, Kocaturk M, Ulus $\mid H$. Choline or CDP-choline attenuates coagulation abnormalities and prevents the development of acute disseminated intravascular coagulation in dogs during endotoxemia. Blood Coagul Fibrinolysis. 2010;21(4):339-48.

24. Ley K, Laudanna C, Cybulsky MI, Nourshargh S. Getting to the site of inflammation: the leukocyte adhesion cascade updated. Nat Rev Immunol. 2007;7(9):678-89.

25. Marechal X, Favory R, Joulin O, Montaigne D, Hassoun S, Decoster B, et al. Endothelial glycocalyx damage during endotoxemia coincides with microcirculatory dysfunction and vascular oxidative stress. Shock. 2008;29(5):572-6.

26. Mulivor AW, Lipowsky HH. Inhibition of glycan shedding and leukocyte-endothelial adhesion in postcapillary venules by suppression of matrixmetalloprotease activity with doxycycline. Microcirculation. 2009;16(8):657-66. 
27. Bansch P, Nelson A, Ohlsson T, Bentzer P. Effect of charge on microvascular permeability in early experimental sepsis in the rat. Microvasc Res. 2011;82(3):339-45.

28. Walther A, Czabanka M, Gebhard MM, Martin E. Glycoprotein IIB/IIA-inhibition and microcirculatory alterations during experimental endotoxemia-an intravital microscopic study in the rat. Microcirculation 2004;11(1):79-88.

29. Yilmaz MS, Yalcin M, Savci V. Cytidine 5'-diphosphocholine restores blood flow of superior mesenteric and renal arteries and prolongs survival time in haemorrhaged anaesthetized rats. Clin Exp Pharmacol Physiol 2006, 33(5-6):415-420

30. Secades JJ, Lorenzo JL. Citicoline: pharmacological and clinical review, 2006 update. Methods Find Exp Clin Pharmacol. 2006;28 Suppl B:1-56.

\section{Submit your next manuscript to BioMed Central and take full advantage of:}

- Convenient online submission

- Thorough peer review

- No space constraints or color figure charges

- Immediate publication on acceptance

- Inclusion in PubMed, CAS, Scopus and Google Scholar

- Research which is freely available for redistribution 